\title{
Studies of fullerenes and carbon nanotubes by an extended bond order potential
}

\author{
Jianwei Che, Tahir Çağın and William A Goddard III \\ Materials and Process Simulation Center, Beckman Institute, California Institute of \\ Technology, Pasadena, CA 91125, USA
}

Received 22 January 1999

\begin{abstract}
We present a novel approach to combine bond order potentials with long-range nonbond interactions. This extended bond order potential consistently takes into account bond terms and nonbond terms. It not only captures the advantages of the bond order potentials (i.e. simulating bond forming and breaking), but also systematically includes the nonbond contributions to energy and forces in studying the structure and dynamics of covalently bonded systems such as graphite, diamond, nanotubes, fullerenes and hydrocarbons, in their crystal and melt forms. Using this modified bond order potential, we studied the structure and thermal properties (including thermal conductivity) of $\mathrm{C}_{60}$ crystal, and the elastic properties and plastic deformation processes of the single-walled and double-walled nanotubes. This extended bond order potential enables us to simulate large deformations of a nanotube under tensile and compressive loads. The basic formulation in this paper is transferable to other bond order potentials and traditional valence force fields.
\end{abstract}

\section{Introduction}

In recent years, properties and structures of nanosize materials have attracted a great deal of attention; because of their unique properties and small dimensionality they have a promising future for applications. Since the discovery of carbon fullerenes [1] and nanotubes [2], a large body of theoretical and experimental work has been focused on the characterization of these materials [3-10]. Significant progress has been made toward understanding the properties and structures of these nanoscale materials. A typical nanoscale system has from tens to millions of atoms. From a computational point of view, the first principle calculations are feasible only for small-size systems; it is prohibitively expensive to carry out calculations using quantum mechanics for large systems (>100 atoms). In this case, classical molecular dynamics simulation is the widely used theoretical method to investigate various mechanical, thermodynamic, and even chemical properties of these systems.

The reliability of molecular dynamics simulation results is mainly determined by the interaction potentials (force fields) used in these calculations. It is crucial to know the validation regime of a certain potential function. In this paper, we will discuss our implementation for empirical bond order potentials and its application to carbon-based nanomaterials. Empirical bond order potentials have a unique advantage over other traditional valence force fields (FF). They are capable of describing the change of chemical bonding in a system. Therefore, they offer an alternative way of investigating chemical reaction mechanisms, especially in a condensed phase. Among various empirical bond order potentials [11-13], the Brenner potential is parametrized specially for various forms of carbon and hydrocarbon systems. It gives correct energetics not only for small gas phase molecules, but also for condensed phase bulk systems (e.g., diamond crystal). Carbon nanotubes, fullerene molecules and carbon clusters (and their crystals) are of particular interest because of their unique structure and properties. Since these materials are all carbon based, it is straightforward to employ the Brenner bond order potential in MD simulations. Robertson et al have used this potential to calculate carbon nanotube energetics and compared the results with LDA calculations [14]. In addition, it has been used for reactions between hydrocarbon species and diamond surfaces [15], and chemical vapour deposition (CVD) of diamond films [13].

In spite of its successes, the current form of empirical bond order potentials, including the Brenner force field, has limitations for accurate calculations of some material properties. One of the serious limitations is the lack of a consistent treatment of long-range interactions, such as van der Waals and Coulomb interactions. For a general condensed phase system, the long-range interactions contribute significantly to material properties. For example, the packing of fullerenes and nanotubes is stabilized through the intermolecular long-range interactions. Consequently, their melting/crystallization temperature, bulk densities and thermal properties are determined mostly by intermolecular long-range interactions.

In this paper, we apply an extended Brenner bondorder dependent potential [16] to the calculation of fullerene 
and nanotube properties. The implementation of long-range interactions in this potential allows the consistent treatment of all types of long-range nonbond (NB) interactions. In addition, it retains all the advantages that bond order potentials have over the traditional valence FF. Moreover, this implementation can also be applied to some traditional valence FF.

The paper is organized as follows: in section 2, we briefly review the basic formulation of Brenner bond order potential and our implementation. The numerical results and discussions are given in section 3 . Section 4 concludes the paper.

\section{Theory}

In the original Brenner bond order potential [13], the total potential energy is expressed as the sum of all pairwise covalent interactions; however, each pairwise interaction implicitly contains the many-body information from its immediate surroundings:

$$
V^{B}=\sum_{i} \sum_{j>i}\left[V_{R}\left(r_{i j}\right)-\bar{B}_{i j} V_{A}\left(r_{i j}\right)\right] .
$$

In the above equation, $V_{R}$ and $V_{A}$ are the repulsive and attractive parts of the pairwise interaction. They have the general Morse potential form [13],

$$
\begin{aligned}
& V_{R}\left(r_{i j}\right)=f_{i j}\left(r_{i j}\right) \frac{D_{i j}^{e} S_{i j}}{S_{i j}-1} \exp \left[-\sqrt{2 S_{i j}} \beta_{i j}\left(r_{i j}-R_{i j}^{e}\right)\right], \\
& V_{A}\left(r_{i j}\right)=f_{i j}\left(r_{i j}\right) \frac{D_{i j}^{e} S_{i j}}{S_{i j}-1} \exp \left[-\sqrt{\frac{2}{S_{i j}}} \beta_{i j}\left(r_{i j}-R_{i j}^{e}\right)\right],
\end{aligned}
$$

where

$$
f_{i j}(r)=\left\{\begin{array}{c}
1, \quad r \leqslant R_{i j}^{(1)}, \\
\left\{1+\cos \left[\frac{\pi\left(r-R_{i j}^{(1)}\right)}{R_{i j}^{(2)}-R_{i j}^{(1)}}\right]\right\} / 2, \\
R_{i j}^{(1)}<r<R_{i j}^{(2)} \\
r \geqslant R_{i j}^{(2)} .
\end{array}\right.
$$

$f_{i j}(r)$ is a cut-off function that smoothly goes to zero at $R_{i j}^{(2)}$. It explicitly restricts the interaction within the nearest neighbours. $\bar{B}_{i j}$ is the bond order parameter [13],

$$
\bar{B}_{i j}=\frac{B_{i j}+B_{j i}}{2}+F_{i j}\left(N_{i}^{(t)}, N_{j}^{(t)}, N_{i j}^{c o n j}\right) .
$$

$B_{i j}$ and $F_{i j}$ are calculated from the coordination information of each pair of atoms, $i$ and $j$. The values for all the parameters are given in Brenner's original paper [13]. It was pointed out earlier that NB interactions are not included in the above formulation. Subsequently, several approaches have been considered to implement the original potential in the presence of NB forces. In the literature, there are two approaches. The first is the switching-on method, and the other is the partition method. The first approach connects the short-range covalent forces with the long-range NB interactions by a smooth function [17]. The latter approach partitions a system into different groups, and only imposes
NB interactions among these distinct groups [18]. Since both approaches have certain limitations, we developed a new scheme to take NB forces into account [16].

In the new implementation, the total potential of a system is given by a combination of valence bond energies and NB interactions,

$$
V^{t o t}=\sum_{i} \sum_{j>i}\left[V_{i j}^{B}+P_{i j} V_{i j}^{N B}\right]
$$

where $V_{i j}$ is simplified notation for $V_{i j}\left(r_{i j}\right)$, and $P_{i j}=P_{j i}$ is a screening function that properly weights the NB contribution in the total energy. It is written as [16],

$$
P_{i j}=f\left(V_{i j}^{B}, V_{i j}^{B}\right) \prod_{k \neq i, j} f\left(V_{i k}^{B}, V_{k j}^{B}\right),
$$

where the superscript $B$ denotes the covalent energy given by the Brenner potential, and $N B$ denotes the long-range NB interactions, and where

$$
f(x, y)= \begin{cases}\exp \left(-\gamma x^{2} y^{2}\right), & \text { if } x<0 \quad \text { and } y<0 \\ 1, & \text { otherwise. }\end{cases}
$$

The force on each atom can readily be evaluated from equation (6) [16]

$$
F_{\alpha \beta}=-\frac{\partial V^{t o t}}{\partial r_{\alpha \beta}} .
$$

As we see from the above formulation, the source and form of NB interactions are not restricted. For instance, it can either be van der Waals or Coulomb interaction, or both. Also, the detailed functional form used in a specific system will not change the above fundamental equations. Therefore, our implementation offers a method with great flexibility. Moreover, it treats all the atoms in the system equivalently and consistently. In the next section, we will apply this method to a few carbon-based nanostructures.

\section{Numerical applications}

As a limiting case, the implemented Brenner potential can also give the correct energetics for systems in which NB interactions do not play a crucial role [16], such as isolated small hydrocarbon molecules. In these cases, the new potential recovers the results of the original Brenner potential. However, since the original Brenner potential does not have NB interactions, it is not possible to stabilize the crystal forms of the molecular systems. Experimental results show that the fullerene $\left(\mathrm{C}_{60}\right)$ crystal has a fcc packing structure. Therefore, it is necessary to include NB interactions. In this paper, we employ a Lennard-Jones 12-6 potential for van de Waals interaction, and the Lennard-Jones parameters were obtained from the first principles calculation [19]:

$$
V_{i j}^{N B}=\epsilon_{0}\left[\left(\frac{\sigma}{r_{i j}}\right)^{12}-2\left(\frac{\sigma}{r_{i j}}\right)^{6}\right] .
$$

Using the extended potential, the $\mathrm{C}_{60}$ fcc crystal can be stabilized. The heat of formation of the $\mathrm{C}_{60}$ fcc crystal is $0.54 \mathrm{kcal} \mathrm{mol}^{-1}$ per atom compared with a free $\mathrm{C}_{60}$ molecule. Figure 1 shows the unit cell obtained from the extended 


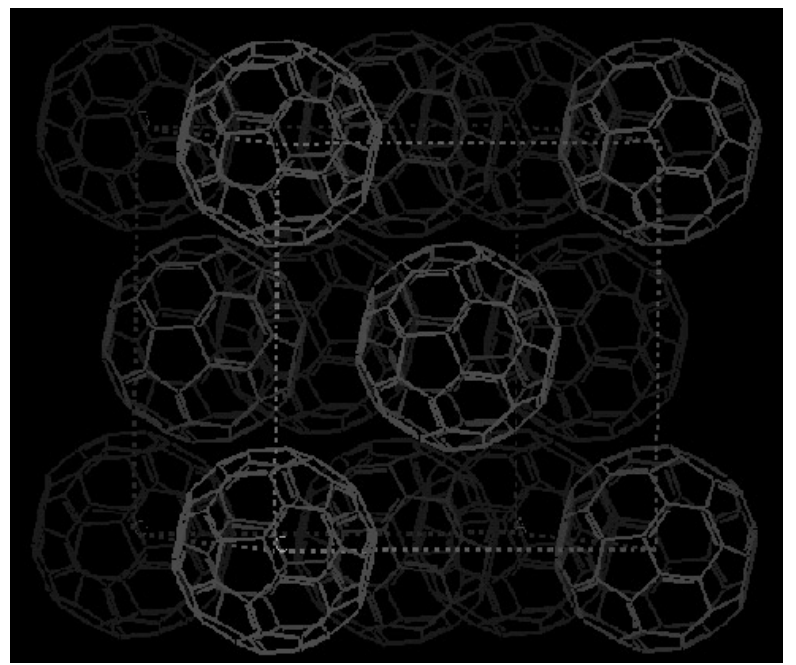

Figure 1. A unit cell of $\mathrm{C}_{60}$ crystal optimized by the extended bond order potential; the calculated lattice constant $a=14.4 \AA$.

Brenner potential. The lattice constant is $14.3 \AA$, which is slightly larger than the experimental value. The discrepancy is the result of inaccurate force constants for covalent bonds rather than the van der Waals forces, because this gives rise to a slightly larger $\mathrm{C}_{60}$ molecule.

With this extended bond order potentials, one can study various transport properties of bucky balls and bucky tubes in condensed phase. Among the transport coefficients, thermal conductivity is one of the most difficult quantities to calculate, especially for solid state materials due to the long mean free path of phonons. However, under certain conditions, the thermal current correlation functions can be evaluated without extending the simulation system. Weakly bonded solids and materials under high temperature have a relatively short phonon mean free path that enables the numerical evaluation of thermal conductivity using rather small simulation cells. In addition to atomistic simulation, the Boltzmann equation for transport phenomena can also be used as a model to calculate the transport properties. Here, we will only demonstrate the feasibility of the extended potential to calculate thermal conductivity through atomistic simulation. To determine the thermal conductivity, we make use of the Green-Kubo relation. The thermal conductivity tensor, $\Lambda$, is given by [20],

$$
\Lambda(T)=\frac{V}{k_{B} T^{2}} \int_{0}^{\infty} \mathrm{d} t\langle\vec{j}(t) \vec{j}(0)\rangle,
$$

where $V$ is the volume of the system and $\vec{j}$ is the thermal current that is written as,

$$
\begin{gathered}
\vec{j}(t)=\frac{\mathrm{d}}{\mathrm{d} t} \sum_{i} \vec{r}_{i}(t) h_{i}(t), \\
h_{i}(t)=\frac{1}{V}\left(\frac{m_{i} v_{i}^{2}}{2}+\frac{1}{2} \sum_{j} u_{i j}\right) .
\end{gathered}
$$

$h_{i}(t)$ is the energy density of site $i$. In our simulation, it represents particle energy density. In general, thermal conductivity is a tensor. For an isotropic material, the

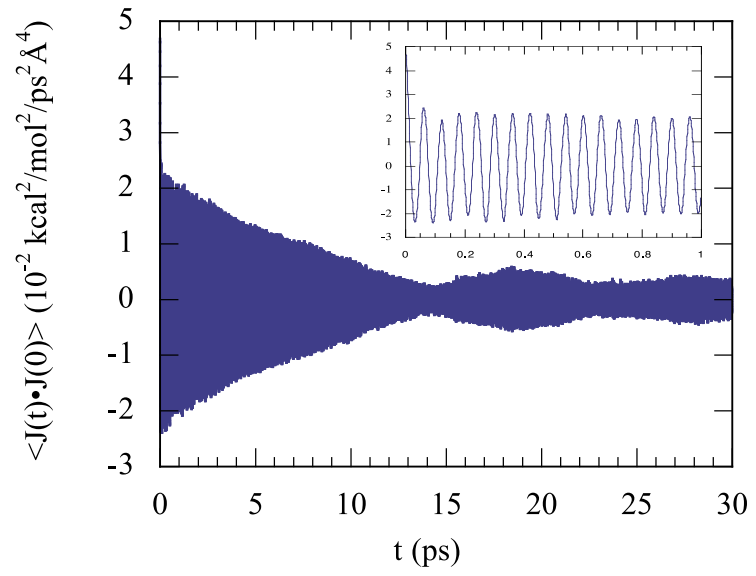

Figure 2. The thermal current-current correlation function at room temperature for the $\mathrm{C}_{60}$ fcc crystal. The inset shows the initial decay of the correlation function.

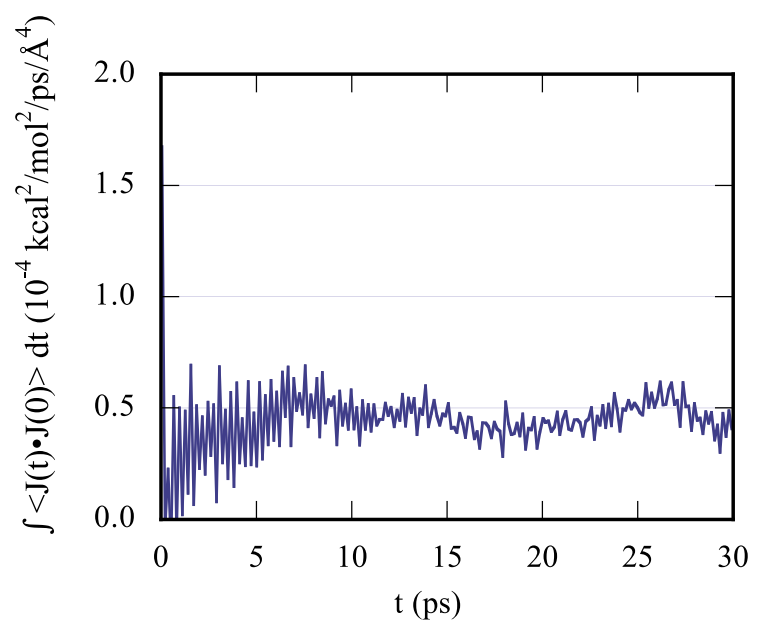

Figure 3. The integration curve for the $\mathrm{C}_{60}$ crystal thermal current correlation function. The final value converges to 0.5 (differing from the thermal conductivity by a constant factor).

average thermal conductivity is measured in experiments. At room temperature, the thermal conductivity of $\mathrm{C}_{60}$ crystal is $0.4 \mathrm{~W} \mathrm{~m}^{-1} \mathrm{~K}^{-1}$. Experiments also showed that the thermal conductivity of $\mathrm{C}_{60}$ crystal is nearly independent of temperature up to $260 \mathrm{~K}$ [21]. In figure 2, we show the thermal current autocorrelation function for $\mathrm{C}_{60}$ crystal at room temperature. The average thermal conductivity is obtained from the integration of the correlation function,

$$
\lambda(T)=\frac{V}{3 k_{B} T^{2}} \int_{0}^{\infty} \mathrm{d} t\langle\vec{j}(t) \cdot \vec{j}(0)\rangle .
$$

The integration curve is depicted in figure 3 . The integration converges to a constant value with a certain fluctuation arising from the fluctuation of the correlation function. The calculated thermal conductivity for $\mathrm{C}_{60}$ at room temperature is $0.4 \mathrm{~W} \mathrm{~m}^{-1} \mathrm{~K}^{-1}$ with a fluctuation of $20 \%$.

In addition to the crystal packing of nanotubes, the determination of accurate energetics of a single-walled nanotube (SWNT) also requires $\mathrm{NB}$ interactions to be considered. Both theoretical predictions and experimental results indicate that nanotubes are among the strongest 


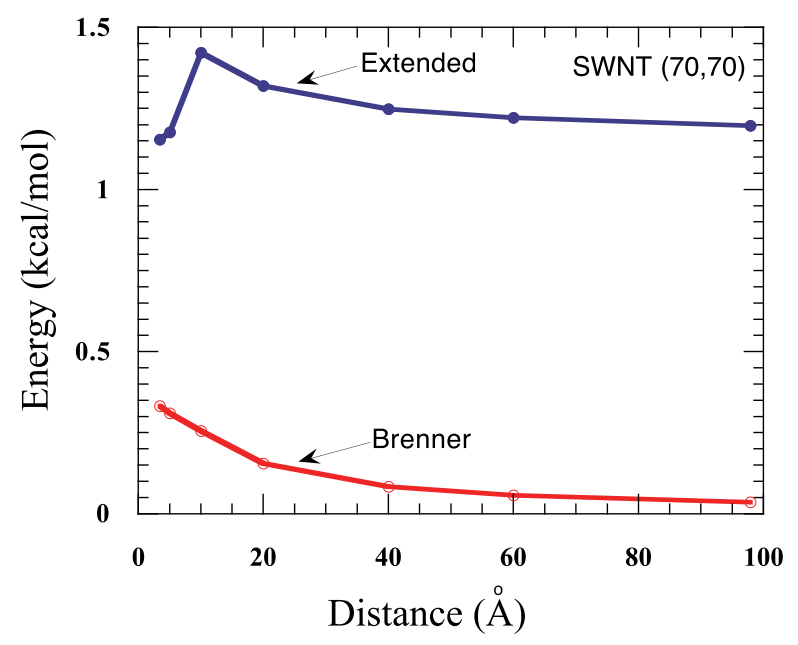

Figure 4. The energetics of a single-walled nanotube $(70,70)$ as a function of the tube conformation. The $x$-axis is the distance of constraints. The solid curve is obtained from the implemented potential, and the dashed curve is calculated from the original Brenner potential.

materials known to date [2]. The pure Brenner potential has been used for studying the structure, energetics, and mechanical properties of SWNT [14]. However, NB interactions can also greatly affect the structures and energetics of SWNT. Both theory [22] and experiment [23] have demonstrated that the most stable shape for large diameter SWNTs is the collapsed form. This is due to the intra-layer van der Waals interaction. Our implementation considers NB interactions within each SWNT and across different tubes for MWNTs. Figure 4 shows the energetics of a $(70,70)$ SWNT as a function of different conformations. For each structure, we applied constraints to restrict the distance between two atoms on the opposite sides across the diameter of the nanotube. Then each structure is optimized under the constraints. The energetics are depicted in figure 4 . The solid curve is obtained from the extended potential, and the dashed curve is calculated from the original Brenner potential. As can be seen from the figure, the extended potential gives the global optimal structure to be a collapsed form, and the circular form is a metastable structure. The original empirical bond order potential only predicts a single optimal structure, which is a circular form. The physical reason for the collapsed structure is that the van der Waals attraction between the two flat area lowers the total energy and localizes the strain energy due to curvature to the opposite ends of the tube cross section. This flat region is similar to the layer stacking in graphite crystal.

For MWNTs, the inclusion of NB interactions is even more important for accurately predicting the material properties. For instance, a double-walled nanotube (DWNT) has an intertube rotational mode. This mode results from the long-range van der Waals interactions. Figure 5 plots the potential as a function of the relative rotational angle between the two nanotubes. The outer tube is a $(15,15)$ SWNT, and the inner one is a $(10,10)$ tube. The space between them is $3.48 \AA$, which is close to the graphite inter layer distance (3.3545 $\AA$ ). This rotational mode is a very low frequency collective mode. When the original form of the Brenner

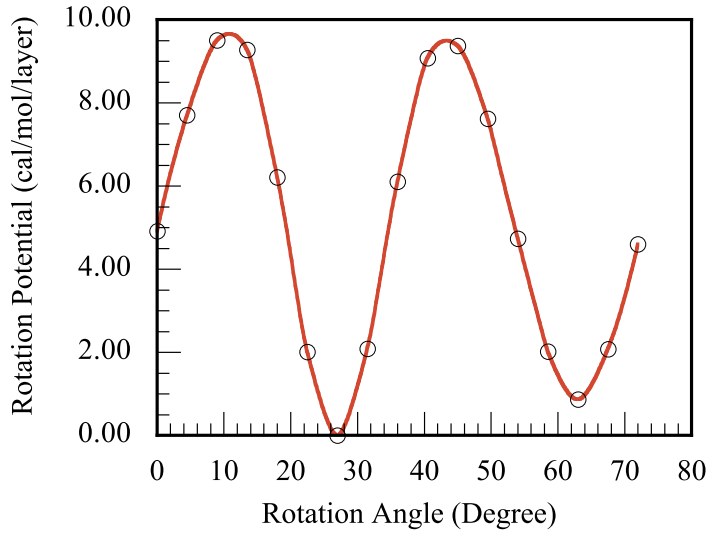

Figure 5. The rotational potential as a function of relative rotational angle between inner and outer tubes. The period for the rotational potential is $72^{\circ}$.

potential is used, the resulting mode is a completely free rotation. However, when NB interactions are considered, one can capture the proper dynamics. From figure 5, the rotational barrier is $10 \mathrm{cal} \mathrm{mol}^{-1}$ per layer. The potential has five periods per one complete rotation, which is the maximum common factor of $(10,10)$ tube and $(15,15)$ tube repeating units.

One of the most important properties of a nanotube is its high strength. Theoretical calculations and experimental results suggest that Young's modulus for a nanotube is on the order of $1 \mathrm{TPa}$. As a comparison, Young's modulus for diamond, the strongest known material, is about $1 \mathrm{TPa}$. In our earlier work [16, 22], we have reported Young's modulus for SWNT and DWNT to be around $940 \mathrm{GPa}$ in the elastic regime. For potential applications of nanotubes, we are interested in the mechanical properties not only in the elastic regime, but also in the plastic regime and the regime where mechanical failure is observed. For a SWNT, the uniaxial stretching mostly involves covalent bond stretching and angle bending. In general, chemical bonds are orders of magnitude stronger than the NB interactions. Therefore, when the deformation may involve chemical bonds, NB forces usually contribute negligibly to the overall deformation. However, for MWNTs, close-packed SWNT bundles, and composite materials, the NB interactions can also be very important where the deformation involves intertube sliding, crossing, and compression. Here, we consider the mechanical behaviour of a SWNT and a DWNT under dynamic stretching. For DWNT, no intertube sliding is allowed. Therefore, NB interactions are not very crucial in the calculation. Here, the partition method can also be employed to include the interlayer NB interactions only. The drawback of this method is that it prevents chemical reactions between atoms that belong to different layers. In our first calculation, a single nanotube of 20 layers long is used. For a $(10,10)$ tube, it contains 800 atoms, and $(15,15) /(10,10)$ DWNT consists of 2000 atoms. One end of the tube is held stationary, and the other end is stretched with a constant velocity (constant strain rate). Figure 6 shows the force and energy of the $(10,10)$ SWNT during the stretching process. The solid curves are calculated from the extended potential, and the dashed curves are obtained from 
the original Brenner potential. From the force evaluation, it is clear that the tube undergoes elastic deformation first and then plastic deformation. The discontinuity occurs when the SWNT suffers a mechanical failure (rupture). As we can see from figure 6 , both the extended and the original Brenner potential give similar results. The extended potential requires a larger force and more energy; this is due to a small potential barrier created by NB interactions before the tube breaks apart. However, the difference is small. More interestingly, figure 6 shows that the force constant increases from $t=12 \mathrm{ps}$ as the system approaches the failure point. At first glance, it is very similar to work-hardening. However, it is caused by the cut-off function $f_{i j}$ in equations (2) and (3). The function artificially increases the force constant while it is turned on from $R_{i j}^{(1)}$ to $R_{i j}^{(2)}$. Physically, this system should approach the failure point of the plastic deformation regime without going through a 'work-hardening' process. This is another shortcoming of the Brenner potential. The cut-off function restricts the covalent interaction within $2 \AA$ (in general-it is not always the case). When a covalent bond is stretched, the two electrons in the bonding orbital are still spin-paired even if the two nuclei are separated by more than $2 \AA$. In other words, the covalent bonding still exists although it is weakened. In order to overcome this problem, a new scheme of calculating bonding information rather than a simple distance criterion is necessary. The same dynamic stretching is also applied to a $(15,15) /(10,10)$ DWNT. Since NB interactions are not important in this process, one can expect that the energy and force are a simple addition of a $(15,15)$ SWNT and a $(10,10)$ SWNT. Figure 8 plots the energy and force as a function of time for the DWNT. Since the stretching is at constant strain rate, the strain is proportional to its timescale. Because the stretching velocity is high, nanotubes do not have time to re-arrange themselves to form defect structures, such as a five membered ring [24]. From figures 6 and 8, the rupture occurs around 18 ps. It is much shorter than the timescale to form a defected structure, which is of the order of a nanosecond [24]. Therefore, this type of dynamic stretching can be considered as an adiabatic process. From the figures, we can also see that the rupture occurs within a very short timescale. Once the tube starts to break, complete breakage happens extremely fast. Figures 7 and 9 show the time evolution of the dynamic stretching processes.

\section{Conclusions}

In this paper, we applied an extended bond order potential to nanotube and fullerene simulation. The extended potential considers NB interactions properly, and offers a generic scheme to implement bond order potential with long-range interactions. Moreover, the modification of bond terms and NB terms can be separable. In other words, one can further improve bond potential or NB potential without worrying too much about the correlation effects. For example, we have discussed the problem of a $2 \AA$ cut-off in bond terms in the above section. To overcome this drawback, one might use other schemes which have much longer bonding range; the $\mathrm{NB}$ terms can remain untouched during the modification. Therefore, this is a robust scheme which

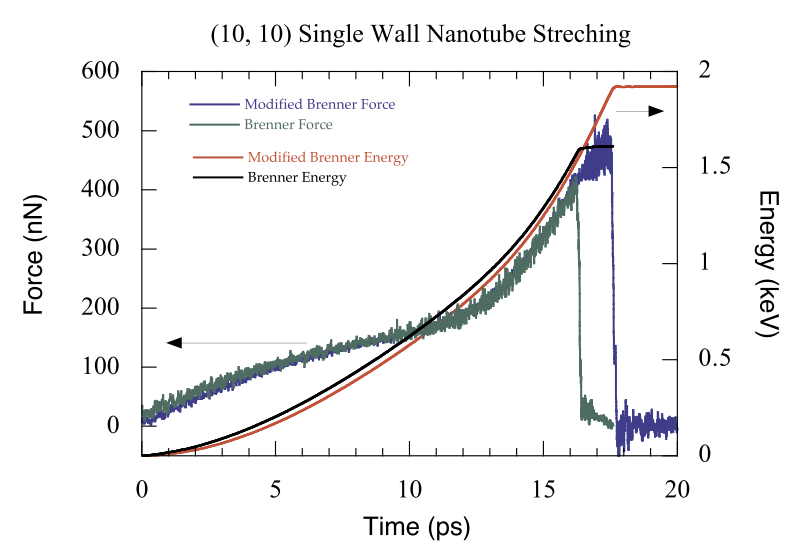

Figure 6. The force and energy as a function of dynamic stretching time for $(10,10)$ SWNT. The solid curve is calculated from the implemented potential, and the dashed curve is computed from the original Brenner potential.

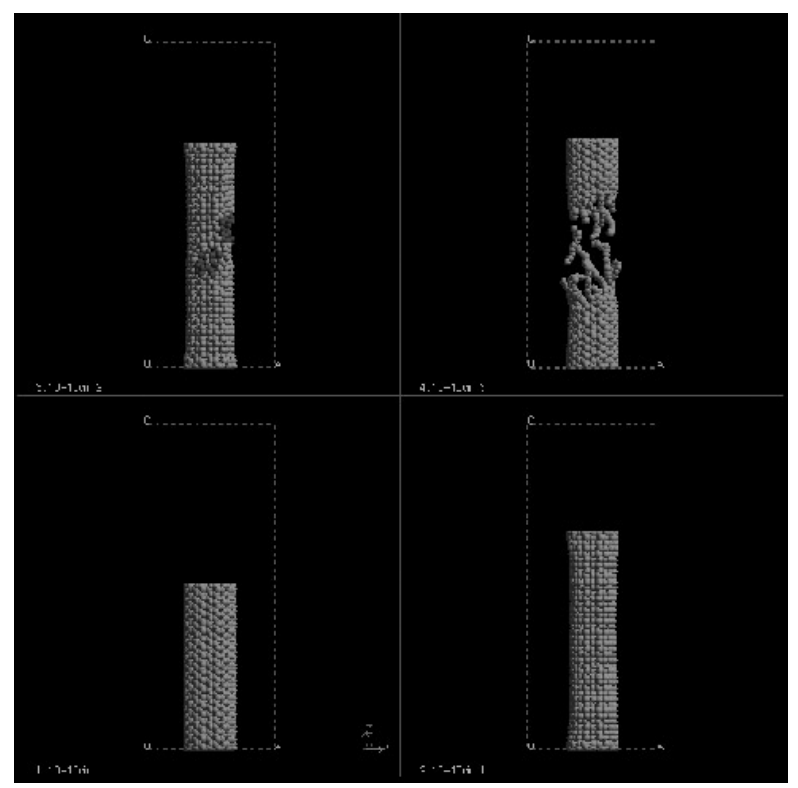

Figure 7. The time evolution of dynamic stretching of $(10,10)$ SWNT.

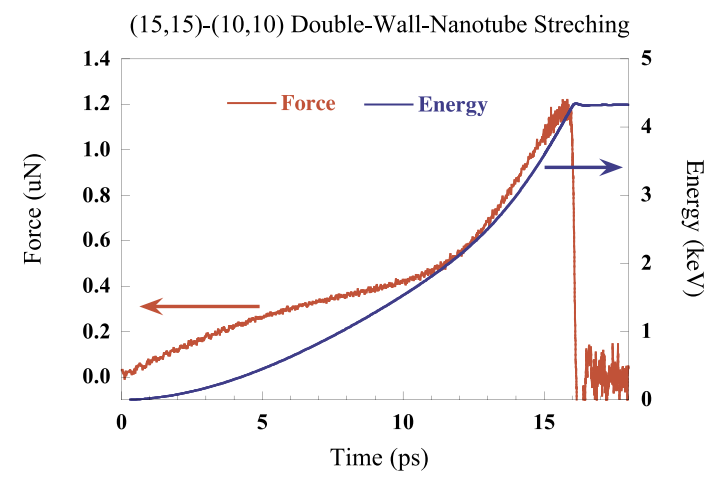

Figure 8. The force and energy as a function of dynamic stretching time for $(15,15) /(10,10)$ DWNT. They are obtained from the implemented potential.

includes NB interactions. For the switching-on method, it needs to be reformulated or re-parametrized significantly if 


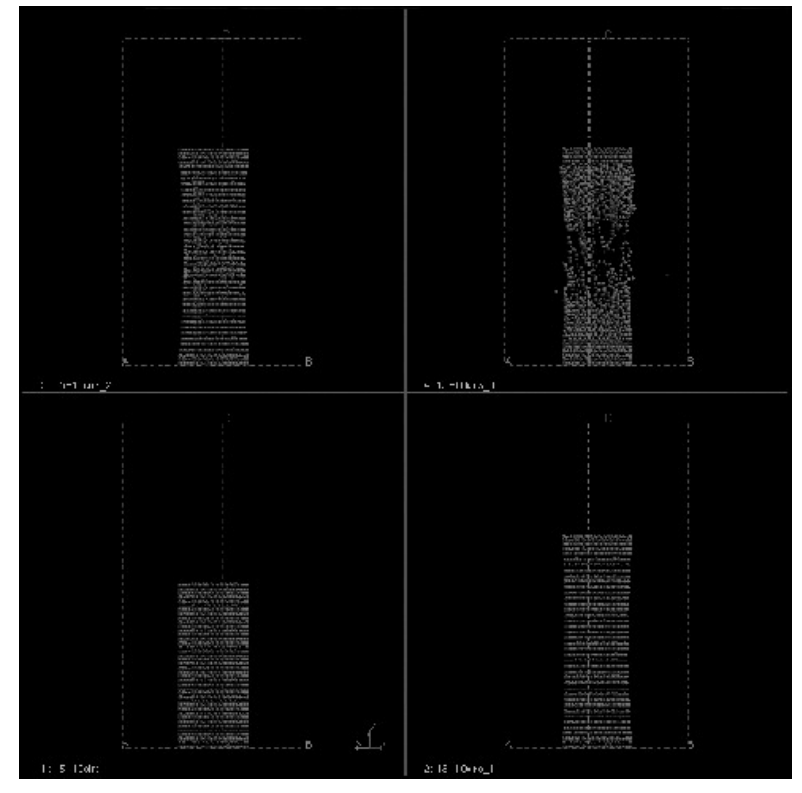

Figure 9. Time evolution of dynamic stretching of $(15,15) /(10,10)$ DWNT. The inner tube $(10,10)$ has been coloured red for clarity.

one alters the bond terms. Using the new extended potential, we also discussed the conditions under which NB interactions are important and the situations where NB terms are not crucial. Through examining the bond order potential in more detail, we pointed out a few shortcomings of the existing potential and the directions that can be taken. We believe that bond order potentials are very powerful tools for classical simulations of material properties.

\section{Acknowledgments}

This research was funded by a grant from NASA (Computational Nanotechnology), and by a grant from DOEASCI. The facilities of the MSC are also supported by grants from NSF (ASC 92-17368 and CHE 91-12279), ARO (MURI), ARO (DURIP), ONR (DURIP), Chevron Petroleum Technology Co., Asahi Chemical, Owens-Corning, Exxon,
Chevron Chemical Co., Asahi Glass, Chevron Research Technology Co., Avery Dennison, BP America, and the Beckman Institute.

\section{References}

[1] Kroto H W, Heath J R, O'Brien S C, Curl R F and Smally R E 1985 Nature 318162

[2] Iijima S 1991 Nature 35456

[3] Treacy M M J, Ebbesen T W and Gibson J M 1996 Nature 381678

[4] Mintmire J W, Dunlap B I and White C T 1992 Phys. Rev. Lett. 68631

[5] Hamada N, Sawada S and Oshiyama A 1992 Phys. Rev. Lett. 681579

[6] Dresselhaus M S, Dresselhaus G and Saito R 1992 Solid State Commun. 84201

[7] Ebbesen T W, Lezec H J, Hiura H, Bennett J W, Ghaemi H F and Thio T 1996 Nature 38254

[8] Lu J P 1997 Phys. Rev. Lett. 791297

[9] Yakobson B I, Brabec C J and Bernholc J 1996 Phys. Rev. Lett. 762511

[10] Dai H, Hafner J H, Rinzler A G, Colbert D T and Smalley R E 1996 Nature 384147

[11] Tersoff J 1986 Phys. Rev. Lett. 56632

[12] Tersoff J 1988 Phys. Rev. Lett. 612879

[13] Brenner D W 1990 Phys. Rev. B 429458

[14] Robertson D H, Brenner D W and Mintmire J W 1992 Phys. Rev. B 4512592

[15] Mowrey R C, Brenner D W, Dunlap B I, Mintmire J W and White C T 1991 J. Phys. Chem. 957138

[16] Che J, Çağın T and Goddard W A III 1998 Therm. Chem. Acta. in press

[17] Brenner D W, Robertson D H, Elert M L and White C T 1993 Phys. Rev. Lett. 702174

[18] Brenner D W, Harrison J A, White C T and Colton R J 1991 Thin Solid Films 206220

[19] Guo Y J, Karasawa N and Goddard W A III 1991 Nature 351 464

[20] Kubo R, Toda M and Hashitsume N 1985 Statistical Physics vol II (Berlin: Springer)

[21] Tea N H, Yu R C, Salamon M B, Lorents D C, Malhotra R and Ruoff R S 1993 Appl. Phys. A 56219

[22] Gao G, Çağın T and Goddard W A III 1998 Nanotechnology 9183

[23] Kiang C H Private communication

[24] Nardelli M B, Yakobson B I and Bernholc J 1998 Phys. Rev. Lett. 814656 